\title{
Dissemination and Adoption of Efficacious Parenting Intervention Programs
}

\author{
Jeff R. Temple
}

Published online: 1 April 2011

(C) Springer Science+Business Media, LLC 2011

In addition to a study of drug resistant skills among understudied adolescents in Mexico, this issue includes three articles on parenting intervention programs. These studies go beyond whether or not parenting programs work to include specific factors related to their efficacy. Together, this compilation of articles spans geography (Australia, Europe, and the United States), settings (community, daycare centers, and primary healthcare facilities), and interventions (Triple P: Positive Parenting Program and PACE: Parenting our Children to Excellence). In addition, these studies use a range of state-of-the-art analytic techniques and methodology including Hierarchal Linear Modeling, Structural Equation Modeling, and technique utilization.

Through research and clinical evidence, we know how critically important parenting practices are in the development of a healthy child. Children who grow up in families characterized by positive affective relationships where adaptive behaviors are supported and maladaptive behaviors are discouraged generally exhibit better psychosocial outcomes than youth raised in less functional households. We also know that parenting intervention programs can and do work (Eyeberg et al. 2008; Sandler et al. 2011). Their short- and long-term benefits include decreased risk

J. R. Temple ( $\square)$

Department of Obstetrics \& Gynecology, University of Texas Medical Branch, Galveston, TX, USA

e-mail: jetemple@utmb.edu of child abuse and participation in risky behaviors, improved academic performance, and improved mental health, to name a few. However, like many efficacious programs designed to promote public health, dissemination, adoption, and utilization remain a struggle. Moreover, increased understanding of how parenting prevention programs affect change, who they work for, and what conditions are optimal for implementation would advance the field.

Begal and Dumas examine the efficacy of a groupadministered behavioral intervention program (i.e., PACE) administered to parents of preschoolers recruited from daycare centers. Their hypothesis that higher levels of parental engagement in the intervention (i.e., session attendance, quality participation) would predict better parent and child outcomes is generally supported. Specifically, more actively engaged parents evidenced less stress and more satisfaction than less engaged parents. Further, actively engaged high-risk parents had less stress, more satisfaction, and a lower risk of child abuse potential than their less engaged high-risk counterparts.

The remaining two studies in this issue examine factors related to the uptake of and engagement in another behaviorally oriented parenting intervention program (i.e., Triple P: Positive Parenting Program). Because of the low participation and high attrition rates of community-based parent training programs, Eisner and Meidert sought to examine factors important to participation at each stage of engagement. Interestingly, they found that parents who were 
difficult to enroll and initially participate (i.e., dual earner families and families with a large number of children) were active participants once they became in engaged. In addition, and consistent with the Begal and Dumas study, the authors found a linear relationship between number of sessions attended and number of techniques used. The study by Turner, Nicholson, and Sanders is among the first to examine the dissemination of a parenting intervention in primary care settings. The authors found that program uptake was influenced by practitioner self-efficacy, which was predicted by the perceived quality and specific aspects of the program. Importantly, this suggests that an efficacious program with empirical support is not enough - they must also be perceived as user-friendly and advantageous to the practitioners on the front lines.

Collectively, these studies show that parenting prevention programs can be implemented in realworld settings by non-researchers. Further, they demonstrate that a key question regarding these programs is not if they work but rather how do we ensure engagement by participants and adoption and utilization by agencies. Though additional research is certainly needed (Sandler et al. 2011), these studies begin to answer this question.

\section{References}

Eyeberg, S. M., Nelson, M. M., \& Boggs, S. R. (2008). Evidence-based psychosocial treatments for children and adolescents with disruptive behavior. Journal of Clinical Child and Adolescent Psychology, 37(1), 215-237.

Sandler, I. N., Schoenfelder, E. N., Wolchik, S. A., \& MacKinnon, D. P. (2011). Long-term impact of prevention programs to promote effective parenting: Lasting effects but uncertain processes. Annual Review of Psychology, 62, 299-329. 